\title{
Note on bias in estimates of the regression coefficient in the analysis of genotype-environmental interaction
}

\author{
HANS-PETER PIEPHO \\ Institut für Pflanzenbau and Pflanzenzüchtung, Christian-Albrechts-Universität, Olshausenstrasse 40, W-2300 Kiel, \\ Germany
}

In this paper, bias of two estimates of the regression coefficient of genotypic response to the environmental index is investigated. It is concluded that both estimates are biased, but that the experimental design can be chosen so as to minimize bias.

Keywords: attenuation effect, environmental index, first-order approximation, Taylor series expansion.

\section{Introduction}

Wright (1976) has identified two sources of bias in the estimation of regression coefficients according to the method of Perkins \& Jinks (1968), in which the independent variable is an environmental index computed as the mean of all genotypes. The first is the well-known attenuation effect (Sprent, 1969), which arises because the independent variable is unknown but estimated with error. Hardwick \& Wood (1972) concluded that there is a tendency to underestimate the absolute value of the regression coefficient. The second bias arises because of a correlation between the response variable, i.e. the genotypic value, and the environmental index, onto which the genotypic value is regressed. Wright (1976) suggests a modified estimate of the regression coefficient which, supposedly, removes both of these biases. The following shows that Wright's estimate is biased. Bias in the estimate by Perkins \& Jinks (1968) is also discussed.

\section{Results and discussion}

The model is

$$
y_{i j k}=\mu+g_{i}+\varepsilon_{j}+b_{k}+f_{i j}+e_{i j k},
$$

where $y_{i j k}$ is the value of the $i j$ th combination of the $k$ th block, $\mu$ is the general mean, $g_{i}$ the effect of the $i$ th genotype, $\varepsilon_{j}$ that of the $j$ th environment, $f_{i j}$ their interaction, $b_{k}$ the $k$ th block effect, and $e_{i j k}$ the error associated with the plot (Wright, 1976). We assume random genotypic and environmental effects. The results do not change, if genotypic effects are fixed provided that we no not impose the constraint $\Sigma_{i} f_{i j}=0$, which is common but not necessary (Searle, 1971; McLean et al., 1991). For the means over all $r$ blocks we have

$y_{i j}=\mu+g_{i}+\varepsilon_{j}+f_{i j}+b .+e_{\mathrm{ij}}$,

where

$e_{i j}=\Sigma_{k} e_{i j k} / r$ and $b .=\Sigma_{k} b_{k} / r$.

We are now interested in the regression of $f_{i j}$ on $\varepsilon_{j}$, which leads to the regression coefficient $\beta_{i}$ proposed by Perkins \& Jinks (1968). Their estimate is biased as pointed out above. Wright's modified estimate is

$\hat{\beta}_{i}^{\prime}=\frac{(m-1)}{(m-2)}\left(W_{i}^{\prime} / \bar{W}-1\right)$

where $m$ is the number of genotypes,

$W_{i}^{\prime}=(m /(m-1))\left(W_{i}-V_{i} / m\right)$, and $\bar{W}=\Sigma_{i} W_{i}^{\prime} / m$.

$W_{i}$ is the observed covariance of values of the $i$ th genotype with the corresponding $y_{. j}$ (the mean of all genotypes in the $j$ th environment), and $V_{i}$ is the observed variance of values $y_{i j}$ of the $i$ th genotype.

Wright's estimate of $\beta_{i}$ is biased, which is a result of errors in the derivation of the expected value of $W_{i}$. This error arises because Wright (1976) does not consider the fact that $f_{i j}$ and $f_{i^{\prime} j}\left(i \neq i^{\prime}\right)$ as well as $f_{i^{\prime} j}$ and $\varepsilon_{j}$ are no longer uncorrelated, if a correlation between interaction and environmental effects is assumed. The derivation of expected values is simplified by writing 
down the regression model explicitly instead of relying on the model without regression coefficients (equation 2), (Wright, 1976).

We now rewrite the model in equation $(1)$ to account for the regression coefficient $\beta_{\mathrm{i}}$. With $f_{i j}=\beta_{i} \varepsilon_{j}+a_{i j}$ and $d_{i j}=a_{i j}+e_{i j}$, one obtains

$y_{i j}=\mu+b .+g_{i}+\varepsilon_{j}+\beta_{i} \varepsilon_{j}+d_{i j}$.

Then, we place the usual constraint $\Sigma_{i} \beta_{i}=0$ on the model, and find

$y_{\cdot j}=\mu+b .+g_{.}+\varepsilon_{j}+d_{\cdot j}$,

where $d_{. j}=\Sigma_{i} d_{i j} / m$ and $g .=\Sigma_{i} g_{i} / m$.

With equations (2) and (3), derivation of expected values is straightforward. We obtain

$E\left(V_{i}\right)=\left(1+\beta_{i}\right)^{2} \sigma_{e}^{2}+\sigma_{d i}^{2}$

where $\sigma_{d i}^{2}$ is the variance of $d_{i j}$ of the $i$ th genotype, and

$$
E\left(W_{i}\right)=\operatorname{COV}_{i}\left(y_{i j}, y_{\cdot j}\right)=\left(1+\beta_{i}\right) \sigma_{\varepsilon}^{2}+\sigma_{d i}^{2} / m \text {. }
$$

Hence,

$$
\begin{aligned}
E\left(W_{i}^{\prime}\right)= & {[m /(m-1)]\left[E\left(W_{i}\right)-E\left(V_{i}\right) / m\right] } \\
= & {[m /(m-1)]\left[\left(1+\beta_{i}\right) \sigma_{\varepsilon}^{2}+\sigma_{d i}^{2} / m-\left(1+\beta_{i}\right)^{2} \sigma_{\varepsilon}^{2} / m\right.} \\
& \left.-\sigma_{d i}^{2} / m\right] \\
= & {[m /(m-1)]\left[\left(1+\beta_{i}\right)-\left(1+\beta_{i}\right)^{2} / m\right] \sigma_{e}^{2} }
\end{aligned}
$$

and

$$
\begin{aligned}
E(\bar{W}) & =[m /(m-1)]\left[\left(1-\left(1+\Sigma_{i} \beta_{i}^{2} / m\right) / m\right] \sigma_{\varepsilon}^{2}\right. \\
& =\left[1-\Sigma_{i} \beta_{i}^{2} / m(m-1)\right] \sigma_{\varepsilon}^{2} .
\end{aligned}
$$

Equations (5) - (7) are in disagreement with the equivalent formulas in Wright (1976), which is a result of the errors mentioned above. So Wright's estimate of $\beta_{i}$ is not unbiased.

In the derivation of an unbiased estimate of $\beta_{i}$, Wright (1976) implicitly makes the assumption that

$E\left(W_{i}^{\prime} / \bar{W}\right)=E\left(W_{i}^{\prime}\right) / E(\bar{W})$.

This assumption is only approximately correct. It is in fact an approximation of the Taylor series expansion of $E\left(W_{i}^{\prime} / \bar{W}\right.$ about $E\left(W_{i}^{\prime}\right) / E(\bar{W})$, in which terms of order higher than 1 are neglected, which adds to the bias in Wright's estimate. It should be scrutinized whether terms of order two and higher are really negligible. On including, for example, terms of order 2 (see Johnson \& Kotz, 1971; Hühn, 1990), one obtains:

$$
\begin{aligned}
E\left(W_{i}^{\prime} / \bar{W}\right) \approx & {\left[E\left(W_{i}^{\prime}\right) / E(\bar{W})\right] } \\
& \times\left\{1-\operatorname{COV}\left(W_{i}^{\prime}, \bar{W}\right) / E(\bar{W}) E\left(W_{i}^{\prime}\right)\right. \\
& \left.+\operatorname{VAR}(\bar{W}) /[E(\bar{W})]^{2}\right\} .
\end{aligned}
$$

It appears to be a complicated task to evaluate $\operatorname{COV}\left(W_{i}^{\prime}, \bar{W}\right)$ and $\operatorname{VAR}(\bar{W})$, so we have not pursued this problem further. The use of the Taylor series expansion is in itself a potential source of bias.

An error is also present in Wright's (1976) formula for the expected value of $V_{x}$, the observed variance of the environmental index $y_{. j}$. We find

$$
\begin{aligned}
E\left(V_{x}\right) & =\operatorname{VAR}\left(y_{\cdot j}\right)=\sigma_{\varepsilon}^{2}+\operatorname{VAR}\left(m^{-1} \Sigma_{i} d_{i j}\right) \\
& =\sigma_{\varepsilon}^{2}+m^{-2} \Sigma_{i} \sigma_{d i}^{2}=\sigma_{\varepsilon}^{2}+m^{-1} \sigma_{d}^{2}
\end{aligned}
$$

where $\sigma_{d}^{2}=\Sigma_{i} \sigma_{d i}^{2} / m$. With Wright, we use the approximation

$$
E\left(W_{i} / V_{x}-1\right) \approx E\left(W_{i}\right) / E\left(V_{x}\right)-1 \text {. }
$$

Again, this is only a first-order approximation by Taylor series expansion of $E\left(W_{i} / V_{x}\right)$ about $E\left(W_{i}\right) /$ $E\left(V_{x}\right)$, and it should be specified as such. More accurate results could be obtained by including terms of higher order. With the first-order approximation, we find

$$
\begin{aligned}
E\left(W_{i} / V_{x}-1\right) & \approx \frac{\left(1+\beta_{i}\right) \sigma_{\varepsilon}^{2}+\sigma_{d i}^{2} / m}{\sigma_{\varepsilon}^{2}+m^{-1} \sigma_{d}^{2}}-1 \\
& =\frac{\left(1+\beta_{i}\right) \sigma_{\varepsilon}^{2}+\sigma_{d i}^{2} / m-\left(\sigma_{\varepsilon}^{2}+m^{-1} \sigma_{d}^{2}\right)}{\sigma_{\varepsilon}^{2}+m^{-1} \sigma_{d}^{2}} \\
& =\frac{\beta_{i} \sigma_{\varepsilon}^{2}+m^{-1}\left(\sigma_{d i}^{2}-\sigma_{d}^{2}\right)}{\sigma_{\varepsilon}^{2}+m^{-1} \sigma_{d}^{2}} \\
& =\beta_{i} k_{1}+k_{2}
\end{aligned}
$$$$
k_{1}=\frac{\sigma_{\varepsilon}^{2}}{\sigma_{\varepsilon}^{2}+m^{-1} \sigma_{d}^{2}}=1-\frac{\sigma_{d}^{2}}{m \sigma_{\varepsilon}^{2}+\sigma_{d}^{2}}
$$$$
k_{2}=\frac{m^{-1}\left(\sigma_{d i}^{2}-\sigma_{d}^{2}\right)}{\sigma_{\varepsilon}^{2}+m^{-1} \sigma_{d}^{2}}=\frac{\sigma_{d i}^{2}-\sigma_{d}^{2}}{m \sigma_{\varepsilon}^{2}+\sigma_{d}^{2}} .
$$

Then the bias is

$E\left(\hat{\beta}_{i}\right)-\beta_{i}=\beta_{i} k_{1}+k_{2}-\beta_{i}=\beta_{i}\left(k_{1}-1\right)+k_{2}$.

We see from this equation that there is a bias unless $\beta_{i}=k_{2} /\left(1-k_{1}\right)=\left(\sigma_{d i}^{2} / \sigma_{d}^{2}\right)-1$. Whether this bias is upward or downward depends on the magnitude of $k_{1}$ and $k_{2}$, and therefore on the values of $\sigma_{\varepsilon}^{2}, \sigma_{d}^{2}, \sigma_{d i}^{2}$, and $m$. Generally, the bias will be small when $m$ and $\sigma_{\varepsilon}^{2}$ are large. Theoretically, the bias vanishes as either of these parameters tends to infinity.

If $\sigma_{d i}^{2}=\sigma_{d}^{2}$ for all $i$, as assumed by Hardwick \& Wood (1972), then $k_{2}=0$, and

$E\left(\hat{\beta}_{i}\right)=\frac{\beta_{i} \sigma_{\varepsilon}^{2}}{\sigma_{\varepsilon}^{2}+m^{-1} \sigma_{d}^{2}}=\beta_{i}\left[1-\frac{\sigma_{d}^{2}}{m \sigma_{\varepsilon}^{2}+\sigma_{d}^{2}}\right]$.

And if $m \sigma_{e}^{2} \gg \sigma_{d}^{2}$, then 
$E\left(\hat{\beta}_{i}\right)=\beta_{i}\left[1-\frac{\sigma_{d}^{2}}{m \sigma_{\varepsilon}^{2}}\right]$.

This is equivalent to the approximation by Hardwick \& Wood (1972), when environmental effects are random. From equations (8) and (9) there follows a tendency to underestimate $\beta_{i}$ (Hardwick \& Wood, 1972). The underlying assumption of homogeneous $\sigma_{d i}^{2}$, however, is rather restrictive; and in many situations it is more realistic to allow for heterogeneous variances $\sigma_{d i}^{2}$. In fact, in the analysis of genotype-environmental interaction, $\sigma_{d i}^{2}$ is often considered a parameter of phenotypic stability of the $i$ th genotype (Perkins \& Jinks, 1968; Shukla, 1972), which implies a potential heterogeneity of $\sigma_{d i}^{2}$. In this case the bias of $\beta_{i}$ may be either up or down as discussed above. All these statements are subject to the qualifications that we have used only a first-order approximation of $E\left(W_{i} / V_{x}\right)$.

\section{Conclusion}

It has been shown that Wright's estimate of $\beta_{i}$ does not remove bias as expected (Wright, 1976). It therefore seems impossible to derive an unbiased estimate of $\beta_{i}$ with Wright's approach. This discussion, however, has demonstrated that the potential bias in the estimate by Perkins \& Jinks (1968) can be reduced by increasing the number of genotypes and by selecting a sufficiently diverse set of testing environments.

\section{References}

HARDWICK, R. C. AND WOOD, J. T. 1972. Regression methods for studying genotype-environment interactions. Heredity, 28, 209-222.

HÜHN, M. 1990. Comments on the calculation of mean harvest indices. J. Agron. Crop Sci., 165, 86-93.

JoHnson, N. L. AND KoTZ, S. 1969. Discrete Distributions. John Wiley, New York.

MCLEAN, R. A., SANDERS, w. L. AND STRoup, w. w. 1991. A unified approach to mixed linear models. Am. Stat., 45, 54-64.

PERKINS, J. M. AND JINKS, J. L. 1968. Environmental and genotype-environmental components of variability. III. Multiple lines and crosses. Heredity, 23, 339-346.

SEARLE, S. R. 1971 . Linear Models. John Wiley, New York.

SHUKLA, G. K. 1972. Some statistical aspects of partitioning genotype-environmental components of variability. Heredity, 29, 237-245.

SPRENT, P. 1969. Models in Regression and Related Topics. Methuen, London.

WRIGHT, A. J. 1976. Bias in the estimation of regression coefficients in the analysis of genotype-environmental interaction. Heredity, 37, 299-303. 